\title{
Stokes problem of a convective flow past a vertical infinite plate in a rotating system in presence of variable magnetic field
}

\author{
Mutua, N.M*, Kinyanjui, M.N, Kwanza, J.K \\ Taita Taveta University College \\ Jomo Kenyatta University of Agriculture and Technology \\ Jomo Kenyatta University of Agriculture and Technology \\ *Corresponding author E-mail: nicholasmuthama@ttuc.ac.ke
}

\begin{abstract}
In this paper, Stokes problem of a free convective flow past a vertical infinite plate in a rotating system in presence of variable magnetic field is investigated. The fluid considered is electrically conducting. The equations governing the flow in this case are non-linear, thus they cannot be solved analytically. The finite difference method (FDM) and computer will be employed in solving the non-linear equations. The effects of the various parameters entering into the problem are discussed extensively and are shown graphically. Discussion of results is done by investigating the parameters: $\mathrm{m}$ (the Hall parameter), E (rotational parameter) and $M^{2}$ (The Magnetic parameter). If $G r>0(=0.5)$ then this is plate cooling by free convection currents, while when $G r<0(=-0.5)$, this is plate heating by free convection currents. The effect of a variable magnetic field is to retard the fluid motion by affecting the velocity and temperature.
\end{abstract}

Keywords: MHD Stokes problem, Finite difference method, variable magnetic field, Hall current, free convection.

\section{Introduction}

The study of rotating fluids has had considerable progress in the last few decades. For instance, the effect of an applied variable magnetic field on unsteady free convection flow along a vertical plate has been given considerable interest because of its application in the cooling of nuclear reactors or in the study of the structures of stars and planets. Important engineering applications in which the study of MHD flows of rotating fluids with variable magnetic field poises includes: power generators, heat exchangers, reactors and MHD accelerators among other devices. As such, MHD is used to explain certain phenomena in the universe.

A study on MHD free convective heat and mass transfer of a heat generating fluid past an impulsively started infinite vertical porous plate with Hall current and radiation absorptions was carried out [1]. [2] analyzed MHD stokes free convection flow past an infinite vertical porous plate subjected to constant heat flux with ion-slip current and radiation absorption.

In 2006 [3] studied computational challenges in fluid flow problems, a MHD Stokes problem of convective flow from a vertical infinite plate in a rotating fluid. A study on Hall current effect on Magnetohydrodynamic free convection flow past a semi-infinite vertical plate with mass transfer was done [4]. They discussed the effects of magnetic parameter, Hall parameter and the relative buoyancy force effect between species and thermal diffusion on the velocity, temperature and concentration. The problem of combined heat and mass transfer of an electrically conducting fluid in MHD free convection adjacent to a vertical surface has been analyzed by [5] taking into account the effects of Ohmic heating and viscous dissipation.

An investigation of MHD effect on the flow structure and heat transfer characteristics was carried out [6]. This was studied numerically for a liquid-gas annular flow under a transverse magnetic field. The results showed that temperature distribution in the liquid film and the Nusselt number distribution in the angular direction were influenced by the flow structures with the side walls. [7] studied the effects of Ohmic heating and viscous dissipation on unsteady MHD and slip flow over a porous rotating disk with variable properties in the presence of Hall and Ion-slip currents. In 2003 [8] studied the nonlinear dynamics of traveling waves in rotating Rayleigh-Bernard convection in which he examined the effects of the boundary conditions and of the topology. In 2004 [9] studied the effect of slip velocity on oscillatory MHD flow with radiative heat transfer and variable suction. 
In spite of all these investigations, much has not been done on Stokes problem of a convective flow past an infinite vertical plate in a rotating system in presence of a variable magnetic field. Our present investigation therefore seeks to study Stokes problem of a convective flow past an infinite vertical plate in a rotating system in presence of a variable magnetic field. The aim of the present investigation is to study the effects of a variable magnetic field resulting to Hall currents on MHD stokes problem for a vertical infinite plate in a rotating system. When there is a variable magnetic field, motion of the fluid is decelerated and Hall currents, Hartman numbers become significant and hence their consideration in the analysis has been important.

\section{Mathematical analysis}

In the present study Stokes flow past an infinite vertical plate in rotating system in presence of a variable magnetic field is considered. The magnetic field is applied transversely along the z-axis and perpendicular to the vertical plate. The plate is non-conducting and the fluid is electrically conducting.

At $t>0$, the vertical plate is set into impulsive motion in its own plane (x-axis direction) at a constant velocity $U$.The transverse inhomogeneous magnetic field is in the z-direction. The vertical plate is kept at a lower temperature than the fluid i.e. $T_{\infty}>T_{w}$. Fluid flow is assumed incompressible, Newtonian, electrically conducting and the density fluctuations are Boussinesq approximated. The Boussinesq approximations means that the density differences are confined to the buoyancy term, without violating the assumption of incompressibility and that the effect of the pressure on the fluid density is negligible. The fluid flow being studied is free convectional and takes place along the $\mathrm{x}$-axis under the action of transverse variable magnetic field.

Geometry of the problem

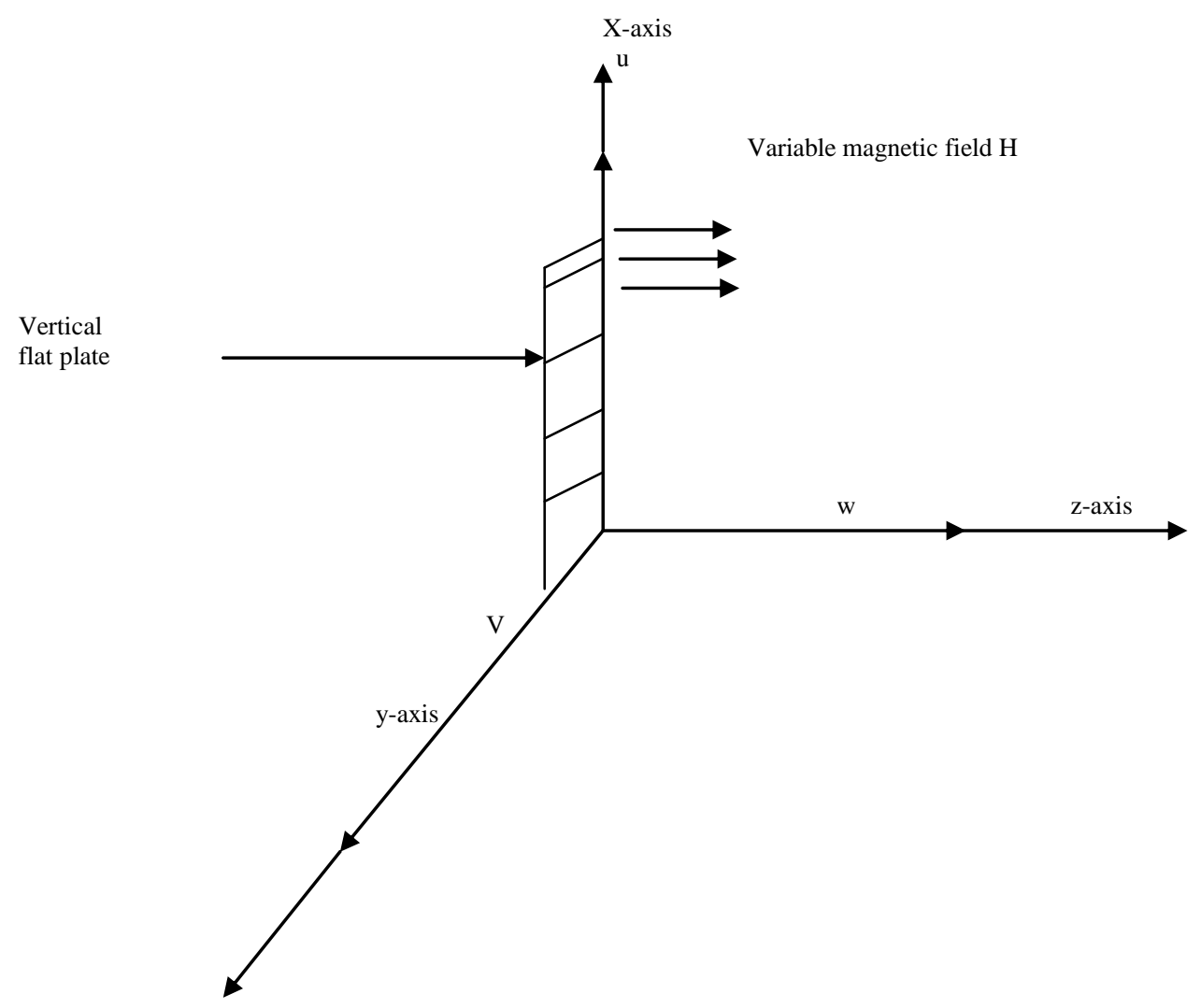

Figure 1: The Flow Configuration with the Coordinate System 
Let the fluid and the plate be in a state of rigid rotation with uniform angular velocity $\Omega$ about the $Z^{*}$ axis taken normal to the plate. In this study the plate is taken to be of infinite length, thus all variables are functions of $Z^{*}$ and $t^{*}$ only. Initially the temperature of the fluid and the plate are assumed to be the same. At time $\mathrm{t}^{*}>\mathrm{O}$, the plate starts moving impulsively in its own plane with velocity $U_{o}$ and its temperature is instantaneously raised or lowered to $T_{w} *$ which is maintained constant. Later on it is assumed that the induced magnetic field is negligible so that $H=\left(0,0, H_{z}\right)$ an assumption which is justified when the magnetic Reynolds is very small (Moreau (1990). The equation of electric charge (i.e. equation $\nabla \cdot \vec{J}=0$, gives $J_{z}^{*}=$ Cons $\tan t$, where $\vec{J}=\left(\overrightarrow{J_{x}} *, \vec{J}_{y} *, \vec{J}_{z} *\right)$. This constant is assumed to be zero, since $J_{z} *=0 \quad$ at the plate which is electrically non-conducting, thus $J_{z} *=0$ everywhere in the flow. The generalized ohm's law including the effects of Hall currents and variable magnetic field (Cowling (1957)) is $\vec{J}+\frac{\omega_{e} \tau_{e}}{H_{o}} \vec{J} \times \vec{H}_{z}=\sigma\left[\vec{E}+\mu_{e} \vec{q} \times \vec{H}_{z}+\frac{1}{e \eta_{e}} \nabla \cdot P_{e}\right]$

Where $\sigma$ is the electrical conductivity, $\mu_{e}$ the magnetic permeability, $\omega_{e}$ the cyclotron frequency, $\tau_{e}$ the collision time, $e$ the electric charge, $\eta_{e}$ the number density of electron and $P_{e}$ the electron pressure respectively. In equation (1), $\vec{q}$ denotes the fluid velocity with components $u^{*}, v^{*}$ and $w^{*}$ in the $x *, y *$ and $z^{*}$-axis directions respectively. In equation (1) the effects of ion-slip and thermoelectric are neglected. In our study we only consider a short circuit problem in which the applied electric field $\vec{E}=0$.Under these assumptions expanding equation (1) we have

$$
\left(J_{x} *, J_{y} *\right)+\frac{\omega_{e} \tau_{e}}{H_{o}}\left(J_{y} * H_{o},-J_{x} * H_{o}\right)=\sigma \mu_{e}\left(v * H_{o},-u * H_{o}\right)
$$

Equating the $x *$ and $y *$ components equation (2) yields

$$
\left.\begin{array}{l}
J_{x} *+\omega_{e} \tau_{e} J_{y} *=\sigma \mu_{e} v * H_{o} \\
J_{y} *-\omega_{e} \tau_{e} J_{x} *=-\sigma \mu_{e} u * H_{o}
\end{array}\right\}
$$

Where $m=\omega_{e} \tau_{e}$ is the Hall parameter. Eliminating $J_{x} *$ from (4) we have

$J_{y} *=\frac{\mu_{e} H_{o}}{1+m^{2}}(m v *-u *)$

Similarly on eliminating $J_{y} *$ from (4) we have

$J_{x} *=\frac{\mu_{e} H_{o}}{1+m^{2}}(m u *+v *)$

In equation (2) and (4) electron pressure has been neglected.

In rotating frame of reference the equation of motion including the Coriolis force (i.e. $-2 \Omega \times q$ ) Greenspan (1968), in components form become:

$$
\begin{aligned}
& \frac{\partial u *}{\partial t}-2 \Omega v *=v \frac{\partial^{2} u^{*}}{\partial z *^{2}}+g \beta *\left(T *-T_{\infty} *\right)+\frac{\mu_{e} J_{y} * H_{z}}{\rho} \\
& \frac{\partial u *}{\partial t}+2 \Omega u^{*}=v \frac{\partial^{2} u^{*}}{\partial z *^{2}}-\frac{\mu_{e} J_{x} * H_{z}}{\rho}
\end{aligned}
$$

Where $g$ is the acceleration due to gravity, $\beta^{*}$ the coefficient of volume expansion, $v$ kinematics viscosity and $\rho$ the density of the fluid. On neglecting the energy dissipated as heat, energy equation becomes

$\frac{\partial T *}{\partial t *}=\frac{\kappa}{\rho C_{p}} \frac{\partial^{2} T *}{\partial z *^{2}}$

Where $\kappa$ is the thermal conductivity, $C_{p}$ the specific heat of the gas at constant pressure. In (7) $T *$ and $T \infty *$ denote the temperature in the boundary layer and free stream respectively, $J_{x} *$ and $J_{y} *$ are the current density components and $u *, v *$ are the velocity components in the $x *$ and $y *$ directions respectively.

In our flow problem we first neglect the displacement current. The variable transverse magnetic field induces a current given by 
$\vec{J}=\nabla \times \vec{H}=\nabla \times \frac{\vec{B}}{\mu_{e}}$

Applying the vector cross product rule equation (10) simplifies to

$\nabla \times \mu_{e} \vec{H}=\mu_{e}\left|\begin{array}{ccc}i & j & k \\ \frac{\partial}{\partial x} & \frac{\partial}{\partial y} & \frac{\partial}{\partial z} \\ 0 & 0 & H_{z}\end{array}\right|=\mu_{e}\left(-i H_{y} \frac{\partial\left(H_{z}\right)}{\partial z}+k \frac{\partial H_{y}}{\partial x}\right)=-j \mu_{e} \frac{\partial H_{z}}{\partial x}$

The component of the current in the X-direction vanishes since any derivative with respect to y is equal to zero i.e. $J_{x}=0$. The resultant induced current which is in the $y$-direction can be expressed as

$J_{y}=\frac{\partial H_{z}}{\partial x}$

The Lorentz force is obtained as

$\vec{J} \times \vec{B}=\vec{J} \times \mu e \vec{H}=\left|\begin{array}{ccc}i & j & k \\ 0 & J_{y} & 0 \\ 0 & 0 & H_{z}\end{array}\right|=i \vec{H}_{z} J_{y}$

Substituting $J_{y}$ using equation (12) in equation (13) yields

$\vec{J} \times \vec{B}=\vec{J} \times \mu_{e} \vec{H}=H_{z} \frac{\partial H_{z}}{\partial x} i$

This force which acts on the fluid particles is in the negative X-direction and therefore trying to oppose the flow. From Ohm's law we have;

$$
\left.\begin{array}{rl}
J=\sigma(\vec{V} \times \vec{B}) & =\sigma(u \vec{i}+v \vec{j}) \times B \vec{k} \\
& =\sigma(-B u \vec{j}+B v \vec{i})
\end{array}\right\}
$$

Thus

$J \times B=\sigma B(-u \vec{j}+v \vec{i}) \times B \vec{k}$

$=-\sigma B^{2} u \vec{i}-\sigma B^{2} v \vec{j}$

$J \times B=-\sigma B^{2}(u \vec{i}+v \vec{j})$

Since $\vec{B}=\mu_{e} \vec{H}$

Then

$J \times B=-\sigma \mu_{e}^{2} H^{2}(u \vec{i}+v \vec{j})$

Or in component form we have

$\left.\begin{array}{l}(J \times B)_{x}=-\sigma \mu_{e}^{2} H^{2} u \\ (J \times B) y=-\sigma \mu_{e}^{2} H^{2} v\end{array}\right\}$

The induction equation is modified by substituting the magnetic field intensity $\mathrm{H}$ with the magnetic induction vector $\vec{B}$. We consider that $\vec{B}$ is in the direction of $\mathrm{H}$ and yielding

$\frac{\partial \vec{B}}{\partial t}+(\vec{u} \cdot \nabla) \vec{B}-(\vec{B} \cdot \nabla) \vec{u}=\frac{1}{\sigma \mu_{e}} \nabla^{2} \vec{B}$

Substituting $\vec{B}=\mu_{e} \vec{H}$ in (21) yields

$\frac{\partial \mu_{e} \vec{H}}{\partial t}+(\vec{u} \cdot \nabla) \mu_{e} \vec{H}-\left(\mu_{e} \vec{H} \cdot \nabla\right) \vec{u}=\frac{1}{\sigma \mu_{e}} \nabla^{2} \mu_{e} \vec{H}$ 
$\mu_{e} \frac{\partial \vec{H}}{\partial t}+\mu_{e}(\vec{u} \cdot \nabla) \vec{H}-\mu e(H \cdot \nabla) \vec{u}=\frac{1}{\sigma} \nabla^{2} \vec{H}$
$\mu_{e}\left[\frac{\partial \hat{H}}{\partial t}+(\vec{u} \cdot \nabla) \vec{H}-(\stackrel{\leftarrow}{H} \cdot \nabla) \vec{u}\right]=\frac{1}{\sigma} \nabla^{2} \vec{H}$
$\frac{\partial \vec{H}}{\partial t}+(\vec{u} \cdot \nabla) \vec{H}-(H \cdot \nabla) \vec{u}=\frac{1}{\sigma \mu_{e}} \nabla^{2} \vec{H}$.

On expanding this equation and using the fact that $H=\left(H_{x}, H_{y}, H_{z}\right)=\left(0,0, H_{z}\right)$ and that the fluid flow depends on $\mathrm{z}$ and $\mathrm{t}$ only while the applied magnetic field depends on $\mathrm{x}$ and $\mathrm{t}$, then $u=w=0$ yields

$\frac{\partial H_{z}}{\partial t}+u \frac{\partial H_{z}}{\partial x}=\frac{1}{\sigma \mu_{e}} \frac{\partial^{2} H_{z}}{\partial x^{2}}$

In this study we are considering a fully developed flow, thus in equations (7) to (9) the inertia terms have been neglected, Hermann Schlichting (1968). As a result of this the solution obtained will be true for a short time after the motion started and temperature jump at the wall (i.e. the results are true in the boundary layer).

In this study we are interested with free convection flows only thus together with the condition of no-slip of the fluid at the wall the boundary and the initial conditions of this problem are:

$\left.\begin{array}{l}t^{*} \leq 0, u^{*}=0, T^{*}=T_{\infty}^{*}, \text { at } z^{*}=0 \\ t^{*}>0, u^{*}=u_{o}, v^{*}=0, T^{*}=T_{\infty}^{*}, \text { at } z^{*}=0 \\ t^{*}>o, u^{*} \rightarrow 0, v^{*} \rightarrow 0, T^{*} \rightarrow T_{\infty}^{*}, \text { at } \quad z^{*} \rightarrow \infty\end{array}\right\}$

In order to normalize equations (7) to (9) we introduce the following non-dimensional quantities:

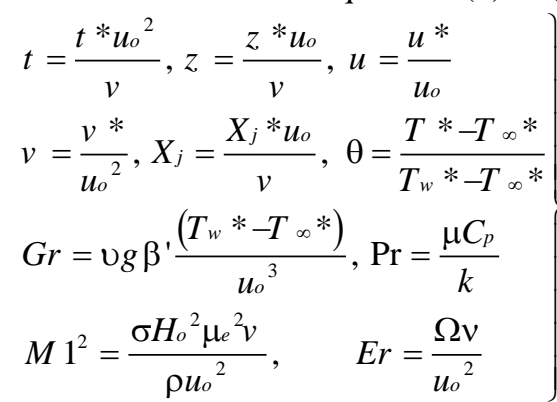

$(28 \mathrm{a}, \mathrm{b}, \mathrm{c}, \mathrm{d})$

Using (28a) equations (7) to (9) become:

$\frac{\partial u}{\partial t}-2 E_{r} v=\frac{\partial^{2} u}{\partial z^{2}}+G_{r} \theta+\frac{M_{1}^{2} H_{z}^{2}}{\left(1+m^{2}\right)}(m v-u)$

$\frac{\partial v}{\partial t}+2 E_{r u}=\frac{\partial^{2} v}{\partial z^{2}}-\frac{M_{1}^{2} H_{z}^{2}}{\left(1+m^{2}\right)}(m u+v)$

$\operatorname{Pr} \frac{\partial \theta}{\partial t}=\frac{\partial^{2} \theta}{\partial z^{2}}$

Multiplying equation (30) by $i,(i)$ is the complex number given by $i=\sqrt{-1}$ ) and adding to (29) we have:

$\frac{\partial \vec{q}}{\partial t}=\frac{\partial^{2} \vec{q}}{\partial z^{2}}+G r \theta-M_{2} \vec{q}$

Where $M_{2}=\left[2 E r i+\frac{m_{1}{ }^{2}}{1+m i}\right]$ and $\vec{q}=u+v i$ is the complex velocity of the fluid.

The induction equation in non-dimensional form become

$\frac{\partial H_{z}^{*}}{\partial t^{*}}+u^{*} \frac{\partial H_{z}^{*}}{\partial x^{*}}=\frac{1}{R_{m}} \frac{\partial^{2} H_{z}^{*}}{\partial x^{* 2}}$

Where $\sigma \mu_{e} L U_{o}=R_{m}$, the Magnetic Reynolds number.

According to Calvert (2002), the electrical conductivity is 'infinite' when $R_{m}$ is large and magnetic effect may be expected to be prominent. If $R_{m}$ is small the magnetic field is not changed appreciably by the flow thus induced magnetic field can be taken to be zero

The boundary conditions (27) in non-dimensional form reduces to 


$$
\left.\begin{array}{l}
t \leq 0, q(z, t)=0, \theta(z, t)=0 \\
t>0, q(0, t)=1, \theta(0, t)=1 \\
t>0, q(\infty, t)=0, \theta(\infty, t)=0
\end{array}\right\}
$$

\section{Method of solution}

To solve equation of motion, energy equation and induction equation for this flow we apply the finite difference method.

Substituting the finite difference form of the partial derivatives (i.e. from equation (31) (32) and (33) respectively) we obtain the following system

$$
\frac{q\left(i^{\prime}, j^{\prime}+1\right)-q\left(i^{\prime}, j^{\prime}\right)}{\Delta t}=\frac{q\left(i^{\prime}+1, j^{\prime}\right)-2 q\left(i^{\prime}, j^{\prime}\right)+q\left(i^{\prime}-1, j^{\prime}\right)}{(\Delta z)^{2}}+\operatorname{Gr} \theta\left(i^{\prime}, j^{\prime}\right)-m_{2} q\left(i^{\prime}, j^{\prime}\right)
$$

And

$$
\operatorname{Pr} \frac{\theta\left(i{ }^{\prime}, j^{\prime}+1\right)-\theta\left(i^{\prime}, j^{\prime}\right)}{\Delta t}=\frac{\theta\left(i{ }^{\prime}+1, j^{\prime}\right)-2 \theta\left(i^{\prime}, j^{\prime}\right)+\theta\left(i^{\prime}-1, j^{\prime}\right)}{(\Delta z)^{2}}
$$

We lastly express the induction equation (26) in implicit finite difference form via time step $H^{i+1}$ considering that the magnetic flux is dependent on $\mathrm{x}$ and $\mathrm{t}$ yielding

$$
\frac{H^{i+1}(i, j)-H_{z}^{i}(i, j)}{\Delta t}+u^{i+1} \frac{H^{i}(i+1, j)-H^{i}(i-1, j)}{2 \Delta x}=\frac{1}{R_{m}} \frac{H^{i+1}(i+1, j)-2 H^{i+1}(i, j)+H^{i+1}(i-1, j)}{(\Delta x)^{2}}
$$

Multiplying through by $R_{m}$ we obtain

$$
\left[\frac{H^{i+1}(i, j)-H_{y}^{i}(i, j)}{\Delta t}+u^{i+1} \frac{H^{i}(i+1, j)-H^{i}(i-1, j)}{2 \Delta x}\right] R_{m}=\frac{H^{i+1}(i+1, j)-2 H^{i+1}(i, j)+H^{i+1}(i-1, j)}{(\Delta x)^{2}}
$$

In equations (35) and (36) the index $i$ 'refers to $z$ and $j$ ' to time. The mesh system in this case is divided by taking $\Delta z=0.1$ and $\Delta t=0.00125$.From equation (34) the initial conditions at $z=0$ takes the form

$q(0,0)=1, \theta(0,0)=1, q\left(i^{\prime}, 0\right)=\theta\left(i^{\prime}, 0\right)=0$, For all except $i^{\prime}=0$

In finite difference the boundary condition $(34 \mathrm{~b})$ takes the form

$$
q\left(0, j^{\prime}\right)=1, \theta\left(0, j^{\prime}\right)=1 \text { For all } j^{\prime}
$$

Though the boundary condition (34c) applies at $z=\infty$, we take $z=4.1$ as corresponding to $z=8$, since both the values of $q$ and $\theta$ tend to zero as $z \rightarrow 4$. Therefore in this section we set $q\left(41, j^{\prime}\right)=\theta\left(41, j^{\prime}\right)=0$ for all $j^{\prime}$. From (35) we note that the velocity at the end of time step $q\left(i^{\prime}, j^{\prime}+1\right), i^{\prime}=1,2, \ldots$ , 40 is computed in terms of velocities and temperatures at points on earlier time step. Similarly, $\theta\left(i^{\prime}, j^{\prime}+1\right)$ is computed from equation (36). The procedure is repeated till $j^{\prime}=400$ i.e. up to time $t=0.5$.During the computation, to test the convergence and stability of the finite difference scheme, computations were made with smaller values of $t$, viz $t=0.0009,0.001$ and 0.0002 .In our analysis we noted that increasing the number of mesh points by using smaller values of $\Delta t$ does not have a significant effect on the result, thus the finite difference scheme used is stable and convergent.

In order to get the physical understanding of this problem and for the purpose of discussing the results, the numerical calculations have been carried out as explained above for both velocity and temperature. In our calculations the Prandtl number is taken to be equal to 0.7 which corresponds to air and magnetic parameter $M_{1}{ }^{2}=5.0$ which signifies strong magnetic field. The calculations were carried out for both $G r>0(=5.0)$ in the presence of cooling of the plate by free convection currents) and $G r<0(=-0.5)$ in the presence of heating of the plate by free-convection currents). Now the results obtained for the unsteady flow for various parameters are displayed in Figures (to be sated after the results).In the next section, a presentation of the numerical method employed in computing the skin friction and the rate of heat transfer at the plate is done. 


\subsection{Calculation of the skin friction and rate of heat transfer}

After obtaining the velocity and temperature distributions of the flow as explained in the previous section we now compute the skin friction given by

$\tau=-\left.\frac{\partial q}{\partial z}\right|_{z=0}$

Where $\tau=\frac{\tau^{*}}{\rho u_{o}^{2}}$.On the other hand the heat flux $\dot{q}$ at the wall is given by

$\dot{q}=-\left.\frac{\partial \theta}{\partial z}\right|_{z=0}$

In order to solve equations (41) and (42) we apply a second-order least squares correlation used over the gradients of the first ten points.

\section{Discussion of results}

We now discuss the behaviour of velocity (both primary $\mathrm{u}$ and secondary $\mathrm{v}$ ), the temperature distribution, the skin friction (average shear stress $\tau_{u o}$ due to primary velocity as well as the shear stress $\tau_{v o}$ due to secondary velocity), for different parameters involved in the flow problem solved in this study.

In order to get physical insight into the problem under study, the velocity field, temperature field, skin-friction and rate of heat transfer are discussed by assigning numerical values to the parameters encountered into the corresponding equations. To be realistic, the values of Eckert number $E c=0.02$ and $E c=0.5$. The value of Prandtl number is chosen as $\operatorname{Pr}=0.71$ that corresponds to air. Grashof number for heat transfer is chosen as $G r=-0.5-0.5$. The values $G r>0$ correspond to cooling of the plate while the values $G r<0$ correspond to heating of the plate. The values of the magnetic parameter $(M=1,1.5)$ and Rotation parameter $(E r=0.05,0.5)$ are chosen arbitrarily.

A program was written and run for various values of velocities and temperatures for the finite difference equations (40), (41) and (43) using different values of Ec, Er, m, t and M. the velocities were classified as Primary velocity (u) and Secondary velocity (v) along the $\mathrm{x}$ and $\mathrm{y}$ axes respectively. The analysis of the data obtained for $\operatorname{Pr}=0.71$ corresponding to air was done and the resultant results are represented graphically on figures 2 to 7 and on tables 1 to 4 . The graphs represent the general trends of the velocities and temperature along the axis of rotation of the flow field. On the other hand, the variation in rate of heat transfer and skin friction on the thermal and velocity boundary layers are depicted by use these tables. A consideration of two cases of heat changes is done. These two cases are cooling at the plate $(\mathrm{Gr}>0(=0.5))$ and heating at the plate $(\mathrm{Gr}<0(=-0.5))$ with constant heat being supplied to or withdrawn from the plate.

Case 1: Cooling at the Plate

In this case, the Grashof number Gr>0. Hence the plate is at higher temperature than the surrounding and so $\mathrm{Gr}=0.5$.

\section{(a) Primary Velocity (u) Profiles}

From figure 2,

(i) An increase in the rotation parameter Er, magnetic parameter $\mathrm{M}_{2}$ and Eckert number Ec leads to a decrease in the velocity profiles. This is because the presence of the transverse magnetic field creates a resistive force similar to the drag force that acts in the opposite direction of the fluid; thus causing the velocity of the fluid to decrease.

(ii) An increase in the Hall parameter $m$ leads to an increase in the velocity profiles.

\section{(b) Secondary Velocity Profiles}

From figure 3, if the magnitude of the velocity is considered it is noted that

(i) An increase in the rotation parameter and magnetic parameter $\mathrm{M}_{2}$ leads to increase in the velocity profiles

(ii) An increase in the Hall parameter and Eckert number leads to a decrease in the velocity profile. The Hall parameter increases with the magnetic field strength. Physically, the trajectories of electrons are curved by the Lorentz force. When the Hall parameter is low, their motion between the two encounters with heavy particles (neutral or ion) is almost linear. But if it is high, the electron movements are highly curved.

\section{(c) Temperature Profiles}

From figure 4 
(i) An increase in the magnetic parameter $\mathrm{M}_{2}$ and an increase in the Eckert number Ec lead an increase in the temperature profile. Increasing the Eckert number causes the fluid the fluid to become warmer and therefore increase its temperature. This is attributed to the viscous dissipation.

(ii) An increase in the Hall parameter $m$ leads to a decrease in temperature profiles. However, as the distance from the plate increases these profiles remain constant.

(iii) An increase in the rotation parameter Er results in no significant change in temperature.

\section{(d) Rate of Heat Transfer}

From table 1

(i) An increase in the rotation parameter Er, Eckert number Ec and magnetic parameter $\mathrm{M}_{2}$ leads to an increase in the rate of heat transfer.

(ii) An increase in the Hall parameter m leads to a decrease in the rate heat transfer.

\section{(e) Skin Friction $\left(\tau_{x}\right)$ along the $x$ axis and $\left(\tau_{y}\right)$ along the $y$-axis}

From table 3

i. An increase in the rotation parameter Er leads to an increase in $\left(\tau_{x}\right)$ but a decrease in $\left(\tau_{y}\right)$. This reduction is due to the increase in the momentum, thermal and magnetic boundary layer thickness which in turn are caused by the deceleration of the magnetic field.

ii. An increase in the Hall parameter $m$ leads to a decrease in both $\left(\tau_{x}\right)$ and $\left(\tau_{y}\right)$. The magnetic field gives rise to a resistive force and slows down the movement of the fluid.

iii. An increase in the Eckert number Ec leads to an increase in $\left(\tau_{x}\right)$ but a decrease in $\left(\tau_{y}\right)$.

iv. An increase in magnetic parameter $\mathrm{M}_{2}$ leads to a decrease in both $\left(\tau_{x}\right)$ and $\left(\tau_{y}\right)$.

Case 2. Heating at the Plate

In this case the Grashof number $\mathrm{Gr}<0$. In this case the plate is at a lower temperature than the surrounding and $\mathrm{Gr}=-0.5$.

\section{(a) Primary Velocity (u) Profiles}

From figure 5,

i. An increase in the Hall parameter $m$ leads to an increase in the velocity profiles.

ii. An increase in the rotation parameter $\mathrm{Er}$, an increase in the Eckert number Ec and magnetic parameter $\mathrm{M}_{2}$ leads to a decrease in the velocity profiles. Since the wall moves in opposite direction to that of the free stream, it tends to retard the flow.

\section{(b) Secondary Velocity (v) Profiles}

From figure 6, considering the magnitude of the velocity we note that

i. An increase in the rotation parameter Er and magnetic parameter $\mathrm{M}_{2}$ lead to an increase in the velocity profiles.

ii. An increase in the Hall parameter $m$ and an increase in the Eckert number Ec leads to a decrease in the velocity profiles. Inclusion of Hall parameter decreases the resistive force imposed by the magnetic field due to its effect in reducing the effect conductivity.

\section{(c) Temperature Profiles}

From figure 7 ,

i. An increase in the magnetic parameter $M$ and an increase in Eckert number Ec lead to an increase in the temperature profiles. The increase in the fluid temperature induces more flow in the boundary layer causing the velocity of the fluid there to increase. The magnetic field produces a huge increment in the magnitude of the temperature. This can be explained physically as follows: it is well known that a magnetic field imparts some rigidity to the conducting fluid. Thus, with increase in the magnetic field, greater effort will be necessary to maintain the rotation of the plate and this implies an increase in temperature with an increase of the parameter M.

ii. An increase in the Hall parameter $m$ leads to a decrease in the temperature profiles. As the distance from the plate increases, these profiles increase.

iii. An increase in the rotation parameter Er has no effect on the change of temperature profiles. 
i. An increase in the rotation parameter Er, Eckert number Ec and magnetic parameter $\mathrm{M}_{2}$ leads to an increase in the rate of heat transfer.

ii. An increase in the Hall parameter m leads to a decrease in the rate of heat transfer.

\section{(e) Skin Friction along $x$-axis and along the $y$-axis}

From table 4, we note that

i. An increase in the rotation parameter Er leads to an increase in $\left(\tau_{x}\right)$ and a decrease in $\left(\tau_{y}\right)$.

ii. An increase in the Eckert number Ec leads to an increase in both $\left(\tau_{x}\right)$ and $\left(\tau_{y}\right)$

iii. An increase in the Hall parameter m leads to a decrease in $\operatorname{both}\left(\tau_{x}\right)$ and $\left(\tau_{y}\right)$. The skin friction in the $y-$ direction is negative since it is in the opposite direction to that of gravitational force.

iv. An increase in magnetic parameter $\mathrm{M}_{2}$ leads to a decrease in $\left(\tau_{x}\right)$ and an increase in $\left(\tau_{y}\right)$.

\section{Conclusion}

Some or all of the parameters affect the primary velocity, secondary velocity and temperature. Consequently their effect alters the rate of heat transfer and skin friction along the $\mathrm{x}$ and $\mathrm{y}$ axes. Increase in $\mathrm{m}$ and Ec leads to an increase in the primary velocity profiles for both free convection cooling and heating at the plate while an increase in the same parameters leads to a decrease in secondary velocity profiles, which is in agreement with [3] , [10] and [7]

Increasing Er, Ec and $\mathrm{M}$ leads to an increase in the temperature profiles for both free convection cooling and free convection heating. Increasing $\mathrm{Er}$ and $\mathrm{M}$ leads to an increase in the rate of heat transfer while increasing $\mathrm{m}$ and Ec results to a decrease in the rate of heat transfer for both free convection cooling and free convection heating. The effect of a variable magnetic field is to retard the fluid motion by affecting the velocity and temperature profiles.

\begin{tabular}{llllll}
\multicolumn{7}{c}{ Cooling at the Plate $(\mathrm{Gr}=0.5)$} & Ec & Er \\
\hline RESULTS & $\mathrm{m}$ & $\mathrm{H}$ & $\mathrm{M}_{2}$ & 0.02 & 0.05 \\
1 TEST & 1.0 & 2 & 24 & 0.02 & 0.05 \\
1 & 2.0 & 2 & 24 & 0.02 & 0.05 \\
2 & 1.0 & 4 & 96 & 0.50 & 0.05 \\
3 & 1.0 & 2 & 24 & 0.02 & 0.50 \\
4 & 1.0 & 2 & 24 & & \\
\hline
\end{tabular}

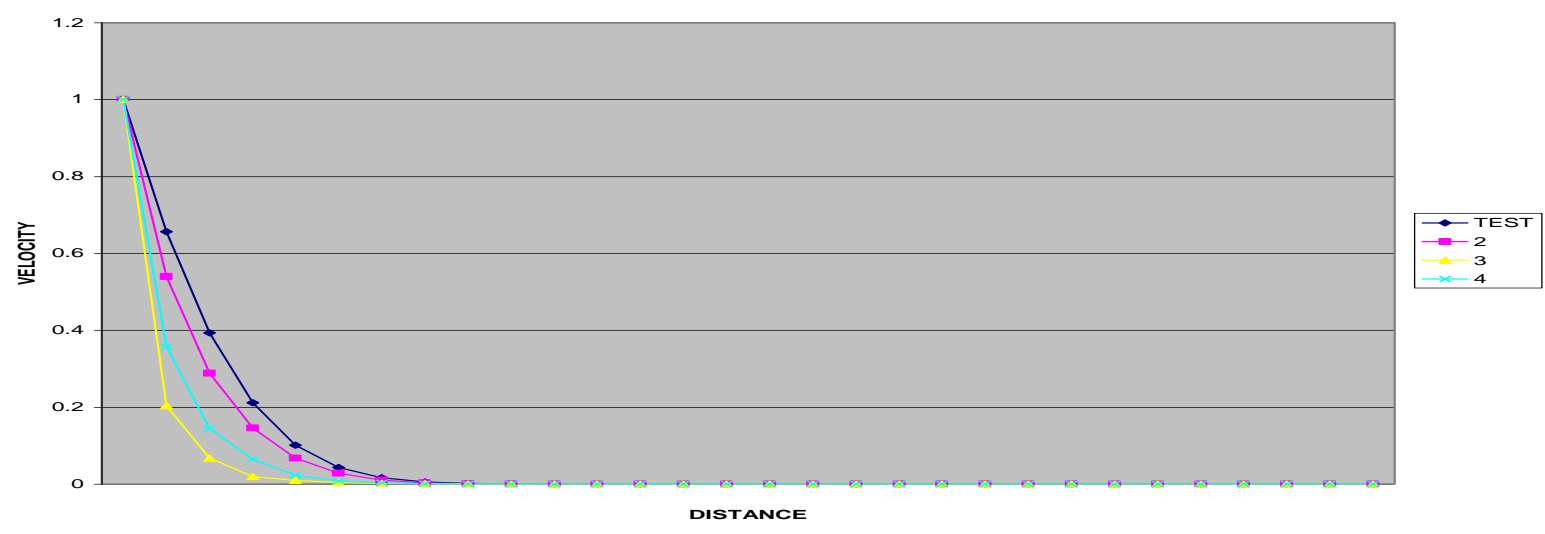

Fig 2: Primary Velocity Profiles (Free Convectional Cooling at the Plate)

\begin{tabular}{llllll}
\multicolumn{7}{c}{ Cooling at the plate $(\mathrm{Gr}=0.5)$} & & Ec & \\
\hline RESULTS & $\mathrm{m}$ & $\mathrm{H}$ & $\mathrm{M}_{2}$ & 0.02 & 0.05 \\
\\
TEST & 1.0 & 2 & 24 & 0.02 & 0.05 \\
1 & 2.0 & 2 & 24 & 0.02 & 0.05 \\
2 & 1.0 & 4 & 96 & 0.50 & 0.05 \\
3 & 1.0 & 2 & 24 & 0.02 & 0.50 \\
4 & 1.0 & 2 & 24 & & \\
\hline
\end{tabular}




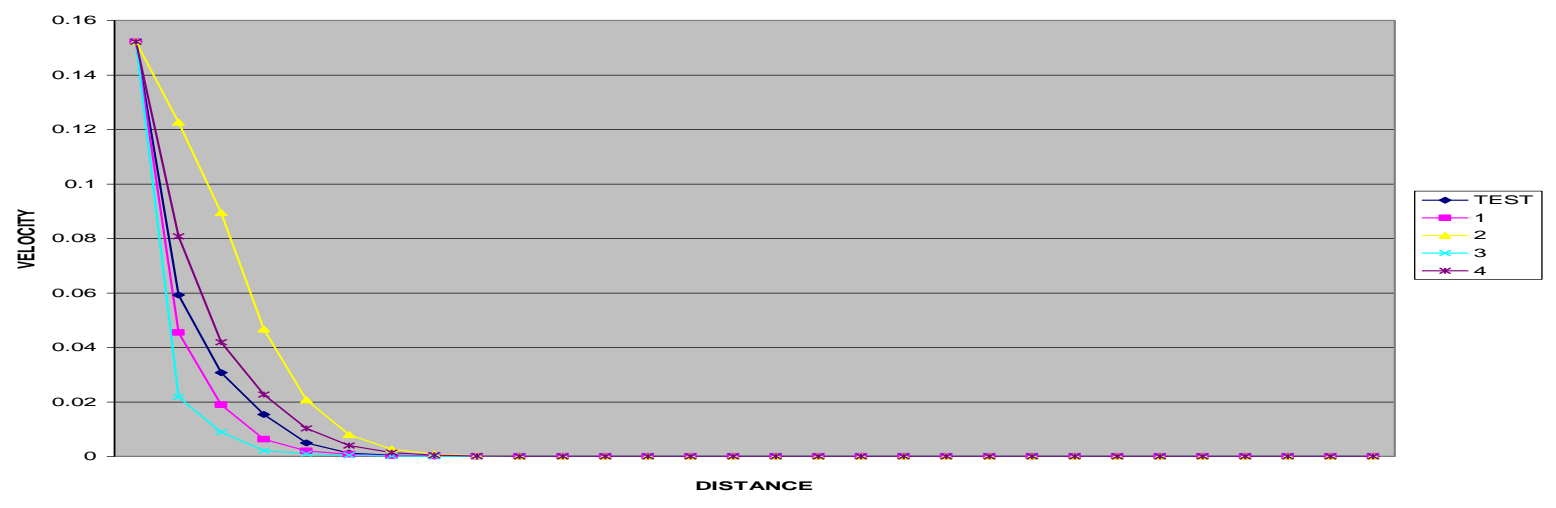

Fig 3: Secondary Velocity Profiles (Free Convectional Cooling at the Plate)

\begin{tabular}{llllll}
\multicolumn{7}{c}{ Cooling at the plate $(\mathrm{Gr}=0.5)$} \\
\hline RESULTS & $\mathrm{m}$ & $\mathrm{H}$ & $\mathrm{M}_{2}$ & $\mathrm{Ec}$ & $\mathrm{Er}$ \\
\hline TEST & 1.0 & 2 & 24 & 0.02 & 0.05 \\
1 & 2.0 & 2 & 24 & 0.02 & 0.05 \\
2 & 1.0 & 4 & 96 & 0.02 & 0.05 \\
3 & 1.0 & 2 & 24 & 0.50 & 0.05 \\
4 & 1.0 & 2 & 24 & 0.02 & 0.50 \\
\hline
\end{tabular}

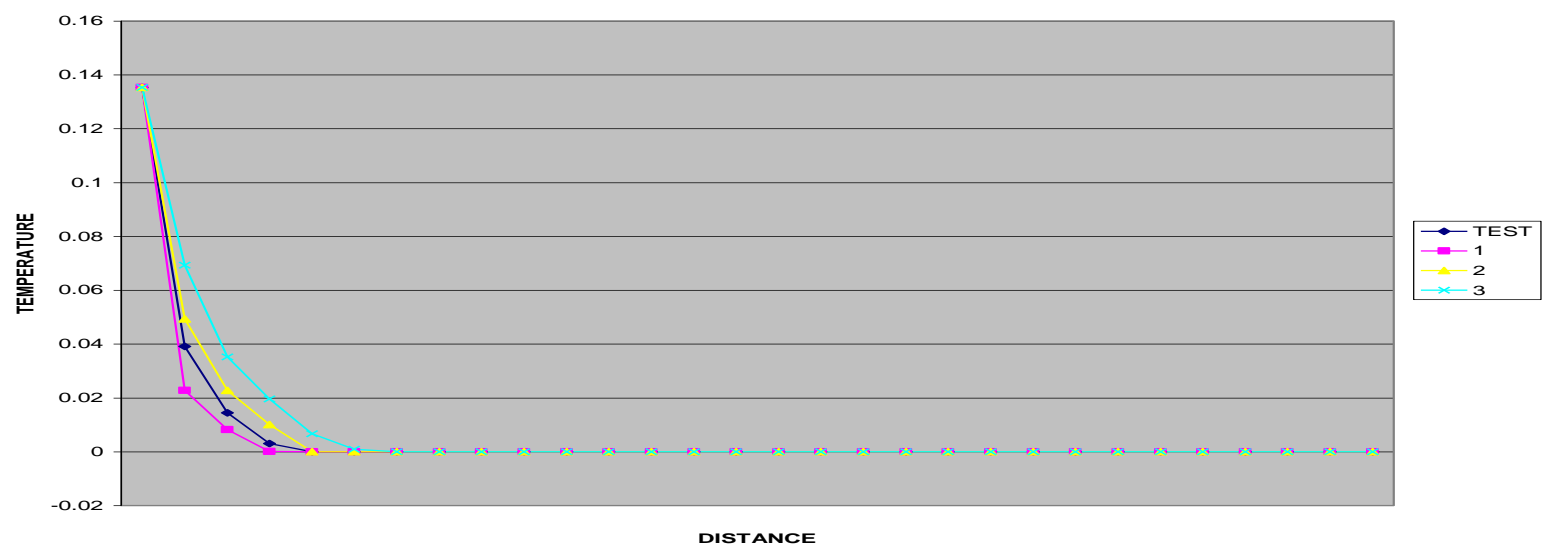

Fig 4: Temperature Profiles (Free Convectional Cooling at the Plate)

\begin{tabular}{llllll}
\multicolumn{7}{c}{ Heating at the plate $(\mathrm{Gr}=-0.5)}$. \\
\hline RESULTS & $\mathrm{m}$ & $\mathrm{H}$ & $\mathrm{M}_{2}$ & $\mathrm{Ec}$ & $\mathrm{Er}$ \\
\hline TEST & 1.0 & 2 & 24 & 0.02 & 0.05 \\
1 & 1.5 & 2 & 24 & 0.02 & 0.05 \\
2 & 1.0 & 4 & 96 & 0.02 & 0.05 \\
3 & 1.0 & 2 & 24 & 0.20 & 0.05 \\
4 & 1.0 & 2 & 24 & 0.02 & 0.50 \\
\hline
\end{tabular}




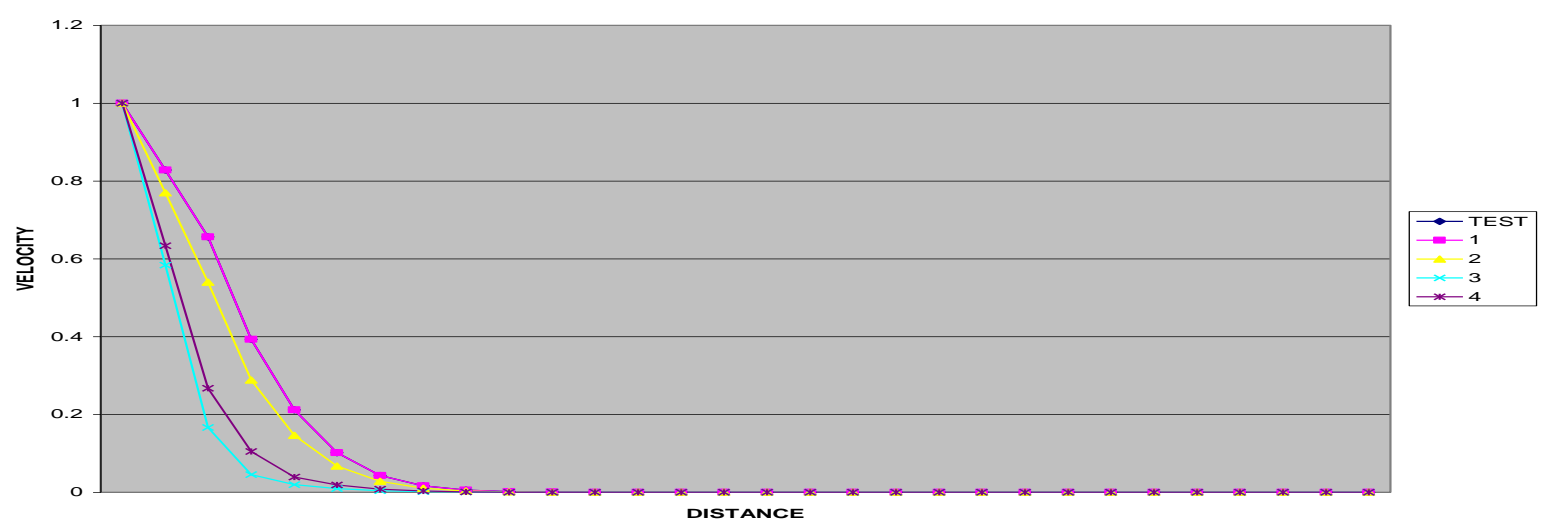

Fig 5: Primary Velocity Profiles (Free Convectional Heating at the Plate)

\begin{tabular}{llllll}
\multicolumn{7}{c}{ Heating at the plate $(\mathrm{Gr}=-0.5)$} & $\mathrm{Ec}$ & $\mathrm{Er}$ \\
\hline RESULTS & $\mathrm{m}$ & $\mathrm{H}$ & $\mathrm{M}_{2}$ & 0.02 & 0.05 \\
1 & 1.0 & 2 & 24 & 0.02 & 0.05 \\
2 & 1.5 & 2 & 24 & 0.02 & 0.05 \\
3 & 1.0 & 4 & 96 & 0.20 & 0.05 \\
4 & 1.0 & 2 & 24 & 0.02 & 0.50 \\
\hline
\end{tabular}

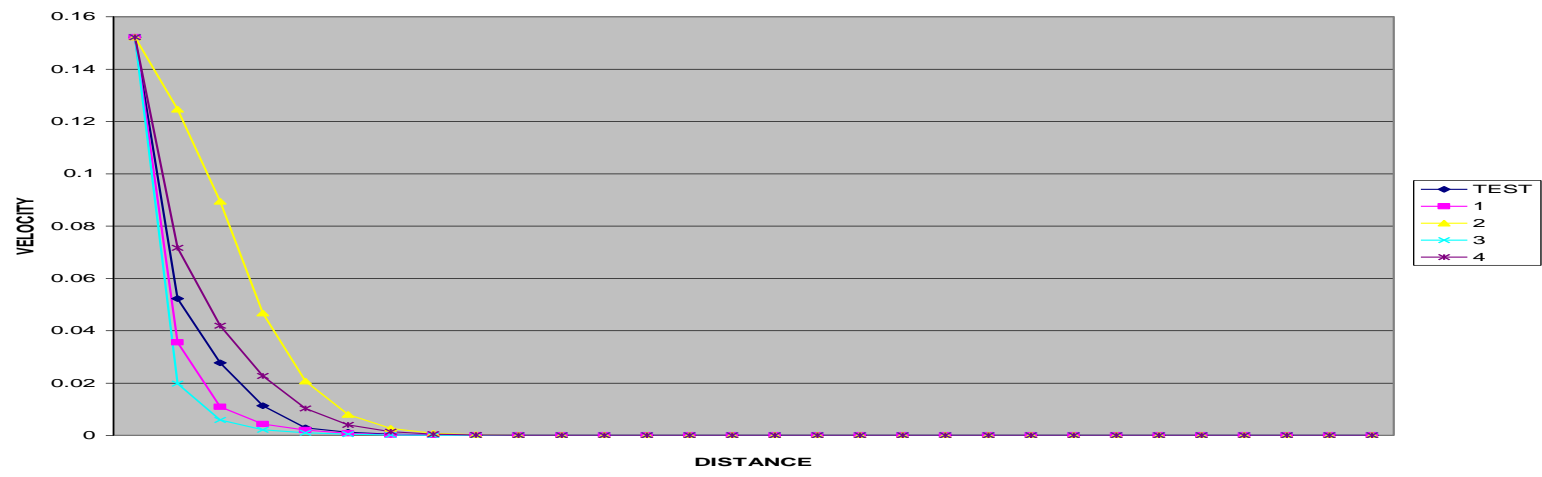

Fig 6: Secondary Velocity Profiles (Free Convectional Heating at the Plate)

\begin{tabular}{llllll}
\multicolumn{7}{c}{ Heating at the plate $(\mathrm{Gr}=-0.5)$} \\
\hline RESULTS & $\mathrm{m}$ & $\mathrm{H}$ & $\mathrm{M}_{2}$ & $\mathrm{Ec}$ & $\mathrm{Er}$ \\
\hline TEST & 1.0 & 2 & 24 & 0.02 & 0.05 \\
1 & 1.5 & 2 & 24 & 0.02 & 0.05 \\
2 & 1.0 & 4 & 96 & 0.02 & 0.05 \\
3 & 1.0 & 2 & 24 & 0.20 & 0.05 \\
4 & 1.0 & 2 & 24 & 0.02 & 0.50 \\
\hline
\end{tabular}




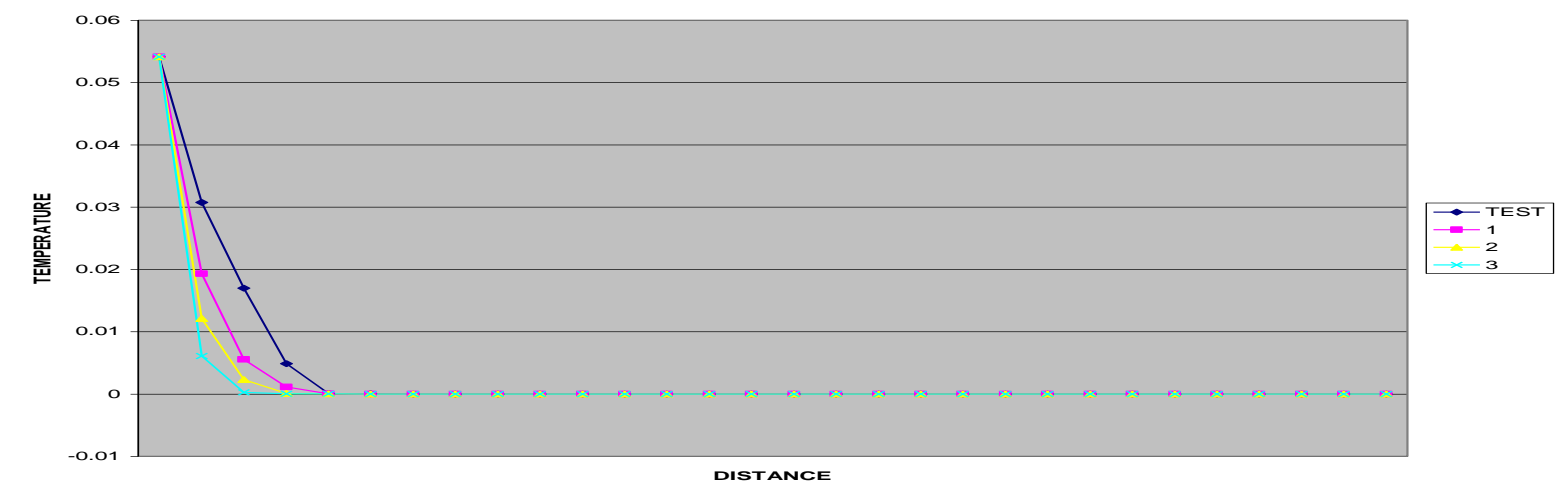

Fig 7: Temperature Profiles (Free Convectional Heating at the Plate)

Table 1: Rate of Heat Transfer With Cooling for $\operatorname{Pr}=0.71$

\begin{tabular}{lllll}
\hline $\mathrm{m}$ & $\mathrm{M} 2$ & $\mathrm{Ec}$ & $\mathrm{Er}$ & $\mathrm{Nu}$ \\
\hline 1.0 & 24 & 0.02 & 0.05 & 1.263899959 \\
2.0 & 24 & 0.02 & 0.05 & 1.263461355 \\
1.0 & 96 & 0.02 & 0.05 & 1.265935278 \\
1.0 & 24 & 0.5 & 0.05 & 1.275017313 \\
1.0 & 24 & 0.02 & 0.5 & 1.263900993 \\
\hline
\end{tabular}

Table 2: Skin Friction $\tau_{x}$ and $\tau_{y}$ With Cooling for $\operatorname{Pr}=0.71$

\begin{tabular}{llllll}
\hline $\mathrm{m}$ & $\mathrm{M} 2$ & $\mathrm{Ec}$ & $\mathrm{Er}$ & $\tau_{x}$ & $\tau_{y}$ \\
\hline 1.0 & 24 & 0.02 & 0.05 & 3.14224995 & -0.139999317 \\
2.0 & 24 & 0.02 & 0.05 & 3.096609455 & -0.169103684 \\
1.0 & 96 & 0.02 & 0.05 & 3.013413321 & 0.00174227625 \\
1.0 & 24 & 0.5 & 0.05 & 3.155085951 & -0.140024952 \\
1.0 & 24 & 0.02 & 0.5 & 3.14696724 & -0.151650365 \\
\hline
\end{tabular}

Table 3: Rate Of Heat Transfer With Heating for $\operatorname{Pr}=0.71$

\begin{tabular}{lllll}
\hline $\mathrm{m}$ & $\mathrm{M} 2$ & $\mathrm{Ec}$ & $\mathrm{Er}$ & $\mathrm{Nu}$ \\
\hline 1.0 & 24 & 0.02 & 0.05 & 0.71 \\
2.0 & 24 & 0.02 & 0.05 & 1.263899959 \\
1.0 & 96 & 0.02 & 0.05 & 1.263572678 \\
1.0 & 24 & 0.5 & 0.05 & 1.266032283 \\
1.0 & 24 & 0.02 & 0.5 & 1.275138535 \\
\hline
\end{tabular}

Table 4: Skin Friction $\tau_{x}$ and $\tau_{y}$ With Heating for $\operatorname{Pr}=0.71$

\begin{tabular}{|c|c|c|c|c|c|}
\hline $\mathrm{m}$ & M2 & Ec & $\mathrm{Er}$ & $\tau_{x}$ & $\tau_{y}$ \\
\hline 1.0 & 24 & 0.02 & 0.05 & 3.151748888 & -0.13965205 \\
\hline 2.0 & 24 & 0.02 & 0.05 & 3.093358238 & -0.168728416 \\
\hline 1.0 & 96 & 0.02 & 0.05 & 3.010100625 & 0.00203710375 \\
\hline 1.0 & 24 & 0.5 & 0.05 & 3.151910738 & -0.139646217 \\
\hline 1.0 & 24 & 0.02 & 0.5 & 3.143599675 & -0.151287697 \\
\hline
\end{tabular}

\section{Acknowledgement}

We would like to acknowledge the National Council of Sciences and Technology (NCST) for funding this project. 


\section{References}

[1] Kinyanjui, M., Kwanza, J.K. and Uppal, S.M. (2001): MHD free convection heat and mass transfer of a heat generating fluid past an impulsively started infinite vertical porous plate with Hall current and radiation absorption, Energy convers. Mgmt. Vol. 42 Pp. $917-931$.

[2] Kwanza, J.K. Kinyanjui, M. and Uppal, S.M, (2003): MHD Stokes free convection flow past an infinite vertical porous plate subjected to constant heat flux with Ion-slip current and radiation absorption, Far East J. App Math. 12(2) Pp.105-131.

[3] Naroua, H. Slaouti, A. and Takhar, H.S. (2006): Computational Challenges in Fluid Flow Problems: A MHD Stokes Problem of Convective Flow from a Vertical Infinite Plate in a Rotating Fluid, European Journal of Scientific Research, Vol.13 Pp.101-112.

[4] Emad M. Aboedahab, Elsayed M.E, Elbarbary. (2001): Hall current effect on Magnetohydrodynamic free-convection flow past a semi-infinite vertical plate with mass transfer, International Journal of Engineering Science, Vol.39 Pp.1641-1652.

[5] Chien-Hsin Chen. (2004): Combined heat and mass transfer in MHD free convection from a vertical surface with Ohmic heating and viscous dissipation, International Journal of Engineering, 42, Pp.669-713.

[6] Feng-Chen Li, Tomoaki Kunugi, Akimi Serizawa. (2005): MHD effect on flow structures and heat transfer characteristics of liquid metal -gas annular flow in a vertical pipe, International Journal of Heat and Mass Transfer Vol.48 Pp.2571-2581.

[7] Osalusi E. side J. Harris R. (2007): The effects of Ohmic heating and viscous dissipation on unsteady MHD and slip flow over a porous rotating disk with variable properties in the presence of Hall and Ion-slip currents. Int. commun. Heat Mass Transfer. doi;10.1016/j.icheatmasstransfer.2007.05.009.

[8] Plaut, E. (2003): Non-linear dynamics of traveling waves in rotating Rayleigh-Bernad convection; Effects of the boundary conditions and of the topology, Physical Review E, 67,046303.

[9] Ogulu, A. and Prakash, J. (2004): Effect of Slip velocity on oscillatory MHD flow with radiative heat Transfer and variable suction, TR 87 CT.

[10] Naroua H, Sambo A.S, Ram P.C, Takhar H.S and Slaouti A. (2004): A software tool using finite elements for the solution of fluid flow problems, Nigerian Journal of Renewable Energy, 12:1\&2, pp.64-68.

\section{Appendix}

\section{ROMAN SYMBOLS}

$\vec{E}$

$\vec{F}$

$e$

$L$

$\vec{J}$

$P$

$\mathrm{U}$

$t^{*}$

$\kappa$

$\vec{q}$

$\vec{B}$

$\vec{D}$

$\vec{H}$

$\vec{i}, \vec{j}, \vec{k}$

$u, v, w$

$\vec{F}_{e}$

$g$

\section{NOMENCLATURE}

\section{QUANTITY}

Electric intensity vector $(\mathrm{V} / \mathrm{m})$

Body force vector $(\mathrm{N})$

Unit charge (C)

Characteristic length (m)

Current density vector $\left(\mathrm{Am}^{-2}\right)$

Pressure force vector $\left(\mathrm{Nm}^{-2}\right)$

Characteristic velocity $\left(\mathrm{ms}^{-1}\right)$

Dimensional Time (S)

Thermal conductivity $\left(\mathrm{Wm}^{-1} \mathrm{k}^{-1}\right)$

Velocity vector $\left(\mathrm{ms}^{-1}\right)$

Magnetic field vector $\left(\mathrm{Wbm}^{-2}\right)$

Electric displacement vector $\left(\mathrm{cm}^{-2}\right)$

Magnetic field intensity vector $\left(\mathrm{Wbm}^{-2}\right)$

Unit vectors in the $\mathrm{x}, \mathrm{y}$ and $\mathrm{z}$ directions

Respectively

Components of velocity vector $\mathrm{q}$

Electromagnetic force $\left(\mathrm{kgm}^{-2}\right)$

Acceleration due to gravity $\left(\mathrm{ms}^{-2}\right)$ 


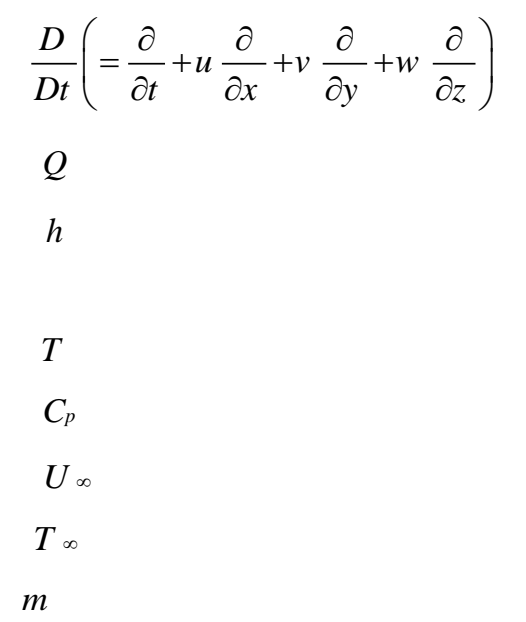

\section{GREEK SYMBOL}

$\mu$

v

$\rho$

$\rho e$

$\sigma$

$\mu e$

$\Delta t, \Delta y, \Delta z$

$\Delta T$

$\nabla$

$\nabla^{2}$

$\varphi$

$\alpha$

$\beta$

$\theta$

$U, V, W$

$x, y, z$
Material derivative

Amount of heat added to the system (Nm)

Dimensional distance between vertical

Plates

General fluid temperature

Specific heat at constant pressure $\left(\mathrm{Jkg}^{-1} \mathrm{~K}^{-1}\right)$

Free stream fluid velocity $\left(\mathrm{ms}^{-1}\right)$

Characteristic free stream temperature $(\mathrm{K})$

Hall parameter

\section{QUANTITY}

Coefficient of Viscosity, $\mathrm{Kg} / \mathrm{ms}$.

Kinematic Viscosity m2s-1

Fluid density, kg/m3.

Electrical charge density (cm-2)

Electrical conductivity $(\Omega-1 \mathrm{~m}-1)$

Magnetic permeability (Hm-1)

Time and distance intervals respectively(s, m)

Temperature change $(\mathrm{K})$

Gradient operator $\left(=i \frac{\partial}{\partial x}+j \frac{\partial}{\partial y}+k \frac{\partial}{\partial z}\right)$

Laplacian operator $\left(=\frac{\partial^{2}}{\partial x^{2}}+\frac{\partial^{2}}{\partial y^{2}}+\frac{\partial^{2}}{\partial z^{2}}\right)$

Viscous dissipation function (s2)

Electrical conductivity

Coefficient of thermal expansion, $\mathrm{K} 1\left[\frac{1}{V}\left(\frac{\partial V}{\partial T}\right)_{p}\right]$

\section{DIMENSIONLESS QUANTITIES}

Dimensionless fluid temperature

Dimensionless fluid velocity

Dimensionless Cartesian coordinates

Dimensionless time 
$E_{c}$

Pr

$R m$

$\mathrm{Nu}$

S

M

Gr

MHD

FDM

HOT
Eckert number $\left\{=\frac{U^{2}}{C_{p}\left(T-T_{\infty}\right)}\right\}$

Prandtl number $\left(=\frac{\mu C p}{k}\right)$

Magnetic Reynolds number $\left(=\sigma \mu_{c} L u\right)$

Nusselt number $\left(=\frac{h L}{\kappa}\right)$

Magnetic force number $\left(=\frac{H o}{L} \frac{\sqrt{\mu c}}{\rho}\right)$

Magnetic Parameter $\left(=\sqrt{\frac{\sigma H o^{2} v}{\mu U_{m}^{2} / v}}\right)$

Grash of number $\left(=\frac{g \beta \Delta T l^{3}}{v^{2}}\right)$

\section{ABBREVIATIONS}

Magnetohydrodynamics

Finite Difference Method

Higher Order Terms 